\title{
Effects of van der Waals forces and salt ions on the growth of water films on ice and the detachment of $\mathrm{CO}_{2}$ bubbles
}

\author{
P. Thiyam ${ }^{1}$, E. R. A. Lima $^{2}$, O. I. Malyi ${ }^{3}$, D. F. Parsons ${ }^{4}$, S. Y. Buhmann ${ }^{5,6}$, C. Persson ${ }^{1,3,7}$ and M. \\ $\operatorname{BOSTRÖM}^{3, a}$ \\ 1 Department of Materials Science and Engineering, Royal Institute of Technology, SE-100 44 Stockholm, Sweden \\ 2 Programa de Pós-graduação em Engenharia Química, Universidade do Estado do Rio de Janeiro, CEP 20550-013, \\ Rio de Janeiro RJ, Brazil \\ 3 Centre for Materials Science and Nanotechnology, University of Oslo, P. O. Box 1048 Blindern, NO-0316 Oslo, \\ Norway \\ 4 School of Engineering and IT, Murdoch University, 90 South St, Murdoch, WA 6150, Australia \\ 5 Physikalisches Institut, Albert-Ludwigs-Universität Freiburg, Hermann-Herder-Str. 3, 79104 Freiburg, Germany \\ ${ }^{6}$ Freiburg Institute for Advanced Studies, Albert-Ludwigs-Universität Freiburg, Albertstraße 19, 79104 Freiburg, Ger- \\ many \\ 7 Department of Physics, University of Oslo, P. O. Box 1048 Blindern, NO-0316 Oslo, Norway \\ a Email corresponding authors: thiyam@kth.se; lima.eduardo@gmail.com; Mathias.Bostrom@smn.uio.no.
}

PACS 34.20.Cf - Interatomic potentials and forces

PACS 87.15.A-- Theory, modeling, and computer simulation

\begin{abstract}
We study the effect of salts on the thickness of wetting films on melting ice and interactions acting on $\mathrm{CO}_{2}$ bubble near ice-water and vapor-water interfaces. Governing mechanisms are the Lifshitz and the double-layer interactions in the respective three-layer geometries. We demonstrate that the latter depend on the Casimir-Polder interaction of the salt ions dissolved in water with the respective ice, vapour and $\mathrm{CO}_{2}$ interfaces, as calculated using different models for their effective polarizability in water. Significant variation in the predicted thickness of the equilibrium water film is observed for different salt ions and when using different models for the ions' polarizabilities. We find that $\mathrm{CO}_{2}$ bubbles are attracted towards ice-water interface and repelled from the vapor-water interface.
\end{abstract}

Brine is known to play a significant role in the dissolution and migration of $\mathrm{CO}_{2}[1]$ during the process of carbon sequestration in deep geological formations 2,3. A number of investigations were performed to understand the role of viscous, capillary, and gravity forces in $\mathrm{CO}_{2}$ storage, and to understand the mechanisms of $\mathrm{CO}_{2}$ movement [4,5]. We are interested in the interaction of $\mathrm{CO}_{2}$ bubbles in water near ice-water and vapor-water interfaces. It is stabilized by repulsive Lifshitz forces 6]. Molecular simulations have also shown that quasi-liquid layers can be formed on ice surfaces 7]. We predict a marked increase in the equilibrium water film thickness on melting ice in the presence of salt ions compared to the case without salt. This could be highly relevant for ice melting (e.g in permafrost and on Mars where transient salt water has been reported [8]).

The interesting aspect of the Lifshitz force is that it can be both attractive and repulsive. This has been understood for a long time 9 11. It was found in the work of Anderson and Sabiski on films of liquid helium on calcium fluoride, and similar molecularly smooth surfaces 12. The films ranged from 10-200 $\AA$ 12]. The thickness of the films could be measured to within a few percent in most cases. For the saturated-film measurements the repulsive van der Waals potential was equal to the negative of the gravitational potential 12. A good agreement was found [11] between these experimental data and the results from Lifshitz theory. In another more subtle experiment Hauxwell and Ottewill $\sqrt{13}$ measured the thickness of films of oil on water near the alkane saturated vapour pressure. For this system, n-alkanes up to octane spread on water. Higher alkanes do not spread. It is an asymmetric system (oil-water-air), and the surfaces are molecularly smooth. The phenomenon depends on a balance of van der 
(i) Wetting film
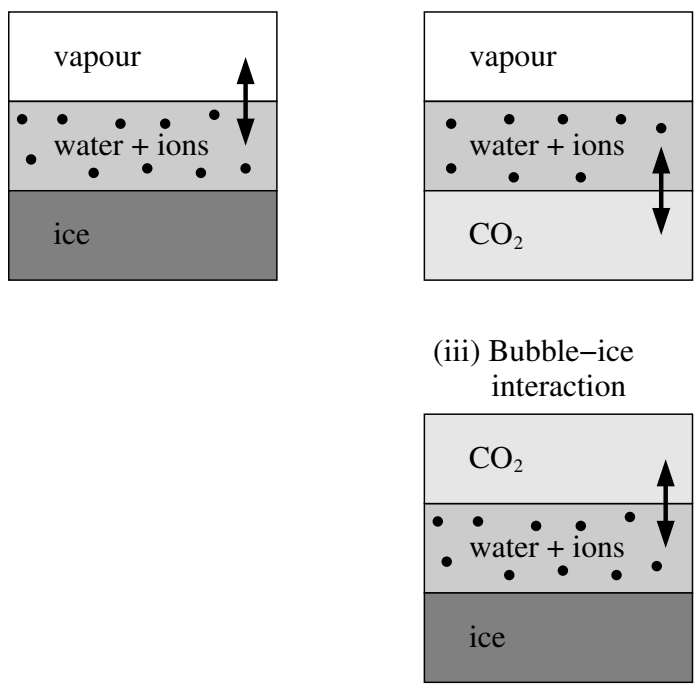

Fig. 1: System under study: (i) The ice--water-vapour system serves to study the film thickness. (ii) The vapour-water- $\mathrm{CO}_{2}$ system approximates a bubble close to the water-vapour interface while (iii) the ice--water- $\mathrm{CO}_{2}$ can be similarly used to study the interaction of the bubble with the water-ice interface.

Waals forces against the vapor pressure $10,13,14$. The net force, as a function of film thickness depends on the dielectric properties of the oils. As demonstrated in ref. [10] it involves an intricate balance of repulsive and attractive components from different frequency regimes. When the ultraviolet and visible components are exponentially damped by retardation the opposing (repulsive) infrared components take over 14.

Our systems of interest are depicted in fig. 1. Our aim is to study the combined effects of the Lifshitz dispersion force and the ion-specific double-layer force on the film thickness and the bubble-interface interactions. To that end, we consider three different relevant planar three-layer subsystems: (i) an ice-water-vapour system can be used to study the film thickness while (ii) vapour-water- $\mathrm{CO}_{2}$ and (iii) ice-water- $\mathrm{CO}_{2}$ systems serve to investigate the interaction of the $\mathrm{CO}_{2}$ bubble in water with the vapour and ice interfaces, respectively. Here, we assume that the bubble is very close to the interface, so that its interaction with the interface can be understood from a planar geometry via the Derjaguin approximation 15$]$.

In the absence of salt, the retarded Lifshitz force turns from short-range repulsion to long-range attraction in the ice-water-vapor subsystem with a transition around 2.1 $\mathrm{nm}$ or $3.6 \mathrm{~nm}$ depending on how the dielectric function of ice is modeled. We use two models ice ${ }_{12}$ and ice $_{13}$ for the dielectric function of ice based on exerimental data in the literature, where the subscript indices indicate the reference numbers listed in ref. 6. In the short range, the double layer interaction adds repulsion to the ice-water- vapor and $\mathrm{CO}_{2}$-water-vapor systems but gives an attractive contribution to the ice-water- $\mathrm{CO}_{2}$ system. This effect from non-linear van der Waals ion-surface forces is in line with the work by Dzyaloshinskii et al. 9, 16, that predicts changes from attraction to repulsion depending on the relative values of the dielectric functions of the three layers comprising the system.

In order to study the effect of salt ions on $\mathrm{CO}_{2}$ bubbles in water and the equilibrium water film thickness at the melting ice surface, we apply different models for the effective polarizability of ions in water. The non-zero effective polarizabilities of ions render ionic dispersion potentials and hence ion-specific concentration profiles between the two uncharged interfaces. This gives rise to a non-linear double-layer contribution $16-19$ that alters the total force between the two interfaces. Note that for two uncharged interfaces there is no double layer interaction in the absence of finite ion polarizabilities. We explore the Lifshitz and double-layer interactions between large $\mathrm{CO}_{2}$ bubbles in water with ice-water or vapor-water interfaces. We discuss how the presence of ions may affect surface sticking of bubbles as well as the equilibrium thickness of Lifshitzrepulsion induced water sheets on ice 6 .

In this study, we consider crystalline $\mathrm{CO}_{2}$ to compute the dielectric function of the $\mathrm{CO}_{2}$ using the Perdew-BurkeErnzerhof functional in density functional theory (DFT). The scissors operator is applied to represent the hybrid functional 21] band gap of $8.4 \mathrm{eV}$. We determine the dielectric functions on the imaginary frequency axis using one version of the Kramers-Kronig dispersion relation [22]. Such an approach was shown to provide resonable description of water dielectric function 23. The Lifshitz and double layer interactions are not very sensitive to the dielectric function of $\mathrm{CO}_{2}$. They are much more sensitive to the dielectric functions of ice and water since these are extremely similar when the water is in equilibrium with the ice. Because of this and since for different phases of a material having similar volumes and electronic properties the dielectric functions are similar 6, 20, it can be speculated that the results predicted in this paper remain qualitatively valid for different $\mathrm{CO}_{2}$ phases (solid, liquid, supercritical). Ice ${ }_{12}$ model is based on energy loss spectroscopy 24 while ice 13 model is based on reflectivity spectrum [25]. As can be observed from fig. 2, the two models give very similar dielectric functions. These dielectric functions are for water and ice at the triple point which assumes zero degrees Celsius and low pressure. However, these dielectric functions have in the past also been used as approximate dielectric functions for water in different phases, at other temperatures and pressures, in a study of van der Waals interactions involving methane gas hydrates (i.e. a mixture of methane and ice/water molecules) [26]. For different ice models, only the inclusion of retardation slightly shifts the point where the Lifshitz force becomes zero. The double layer interaction, on the oher hand, is not so sensitive. The two different interactions are not very sensitive to the dielectric function of $\mathrm{CO}_{2}$ which sup- 


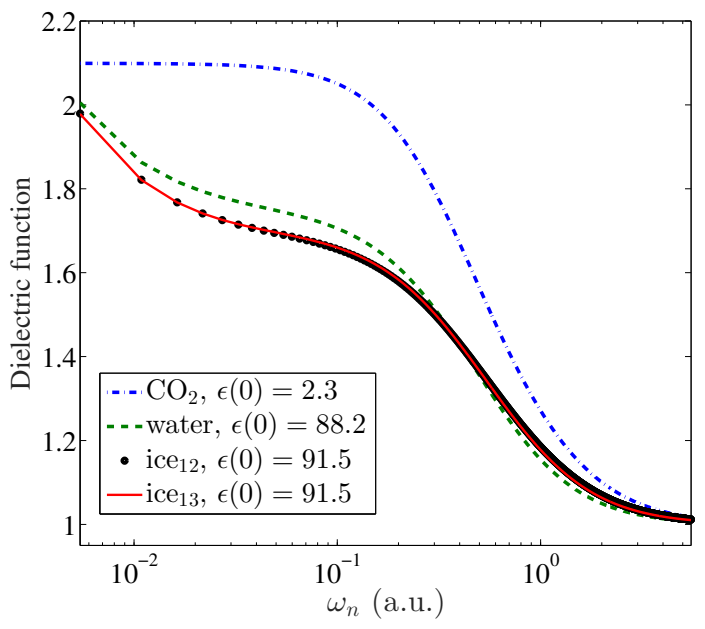

Fig. 2: (Color online) The dielectric functions of $\mathrm{CO}_{2}$, water, ice $_{12}$ and ice $_{13}$ in terms of imaginary Matsubara frequencies. The dielectric constants at zero frequency are also shown in the figure legends.

ports the approximate use of the corresponding crystalline dielectric function.

Using these dielectric functions, the Lifshitz force at temperature $T$ can be calculated following the formalism in the literature 22,27$]$. There is a small correction to the Lifshitz force due to salt ions 27]. However, the effect of screening on the fluctuations due to salt ions on the zero frequency term in the Lifshitz force is very small when we consider salt concentrations around 1-30 $\mathrm{mM}$ and water sheet thicknesses around 1-30 $\mathrm{nm}$. Ions in the water layer may also change the frequency-dependent part of the dielectric response of the water layer, and thus may affect directly non-zero frequency contributions to the Lifshitz force. However, at the very low concentrations studied, the change in dielectric function is very small. Frequency intervals where the intervening medium has a dielectric permittivity in between the permittivity of the two surfaces holding the medium give a repulsive contribution; other intervals give an attractive contribution. The calculation of the force needs accurate dielectric functions over a wide range of frequency for precise predictions.

In order to explore the ion specific double layer interaction in an uncharged three-layer system, we require the dispersion potential between ions and the different interfaces. We use the full theory from Sambale et al. 28 to deduce the simpler non-retarded expressions:

$$
\begin{gathered}
U_{i}(z)=\frac{B_{12}}{z^{3}}+\frac{B_{32}}{(L-z)^{3}} \\
B_{j 2}=\frac{k_{B} T}{2} \sum_{n=0}^{\infty}, \frac{\alpha_{2}\left(i \omega_{n}\right)}{\epsilon_{2}\left(i \omega_{n}\right)}\left(\frac{\epsilon_{2}\left(i \omega_{n}\right)-\epsilon_{j}\left(i \omega_{n}\right)}{\epsilon_{j}\left(i \omega_{n}\right)+\epsilon_{2}\left(i \omega_{n}\right)}\right),
\end{gathered}
$$

for a polarizable particle in the water phase at distances $z$ and $L-z$ from the first and the second interfaces re-

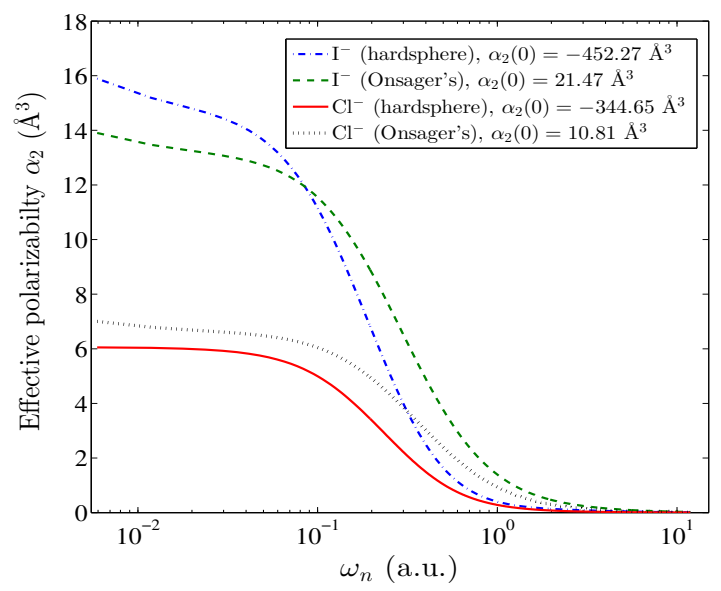

Fig. 3: (Color online) The effective polarizabilities of $\mathrm{Cl}^{-}$and $\mathrm{I}^{-}$for the Onsager and hardsphere models for discrete imaginary frequencies. The zero frequency values are shown in the figure legends.

spectively. In fig. 3, we show illustrative curves for the two models of effective polarizability, viz., the Onsager and the hardsphere models for $\mathrm{Cl}^{-}$and $\mathrm{I}^{-}$ions. These are used to calculate ionic dispersion potentials acting between ions and interfaces. The evaluated $B_{12}$ values used to calculate ionic dispersion potentials are then given in table 1. Here, $k_{B}$ is the Boltzmann constant, $T=273.16$ $\mathrm{K}$ is the temperature, and $\alpha_{2}\left(i \omega_{n}\right)$ is the effective molecular polarizability in water 16 . The prime indicates that the $n=0$ term should be divided by 2. $\epsilon_{1}, \epsilon_{2}$ and $\epsilon_{3}$ are the frequency-dependent dielectric functions of the first material, water and the second material respectively at the imaginary Matsubara frequencies $\left(i \omega_{n}\right)$ given by $\omega_{n}=2 \pi k_{B} T n / \hbar 16,22$.

Two different models are used for the effective polarizability $\alpha_{2}(\omega)$ of the salt ions in water: (i) Onsager's realcavity model for local-field corrections assumes that the particle is inside a small spherical vacuum bubble embedded in the water medium [29]. One finds that 28,30

$$
\alpha_{2}=\alpha\left(\frac{3 \epsilon_{2}}{2 \epsilon_{2}+1}\right)^{2}, \alpha\left(i \omega_{n}\right)=\alpha(0) \sum_{j} \frac{f_{j}}{1+\left(\omega_{n} / \omega_{j}\right)^{2}}
$$

where $\alpha\left(i \omega_{n}\right)$ is the dynamic free-space polarizability 31 . The adjusted parameters $f_{j}$ and $\omega_{j}$ are fitted to agree with the ab initio polarizability in vacuum $\alpha(0)$ by Parsons and Ninham 31. (ii) The hardsphere model posits that the particle is a homogeneous dielectric sphere of radius $a$. The hard sphere ion radii are $a=2.05,2.16,2.33,0.67$, and $1.06 \AA$ for $\mathrm{Cl}^{-}, \mathrm{Br}^{-}, \mathrm{I}^{-}, \mathrm{Na}^{+}$, and $\mathrm{K}^{+} 32$. Its effective permittivity $\epsilon$ can be deduced from the free-space polarizability via 33

$$
\alpha=4 \pi \epsilon_{0} a^{3} \frac{\epsilon-1}{\epsilon+2} .
$$

The excess polarizability of the homogeneous sphere in 


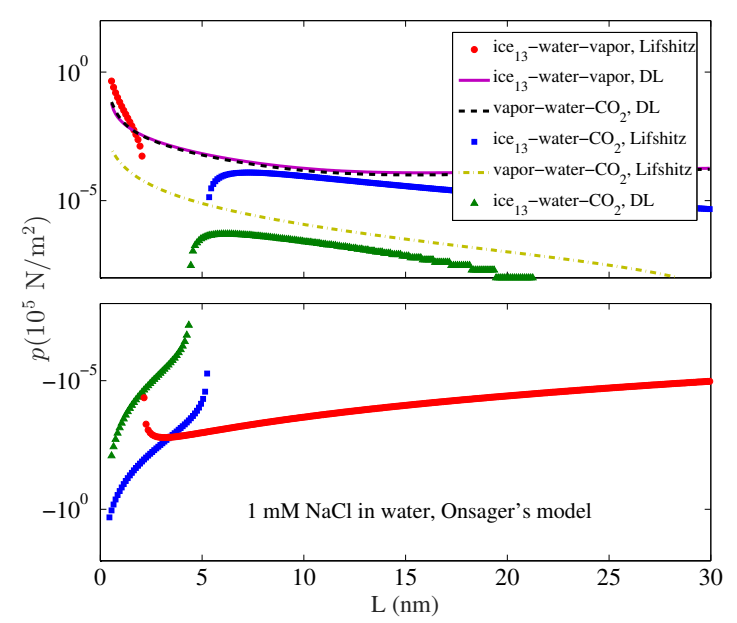

Fig. 4: (Color online) The double layer force between two interfaces separated by $1 \mathrm{mM} \mathrm{NaCl}$, ice-water-vapor, ice-water- $\mathrm{CO}_{2}$ and vapor-water- $\mathrm{CO}_{2}$. The contribution to the total force arising from the Lifshitz energy is also shown. (We use the ice 13 model for the dielectric function of ice and Onsager's model for the effective polarizability of salt ions in water).

water is then 34

$$
\alpha_{2}=4 \pi \epsilon_{0} \epsilon_{2} a^{3} \frac{\epsilon-\epsilon_{2}}{\epsilon+2 \epsilon_{2}} .
$$

To study the specific ion effects, a calculation of the ion profiles between the two interacting surfaces is required. The distribution of ions between the surfaces is determined by a modified Poisson-Boltzmann equation that includes both electrostatic and nonelectrostatic potentials acting on the ions 35

$$
\begin{gathered}
\frac{d^{2} \phi}{d z^{2}}=-\frac{e \sum_{i} z_{i} c_{i, 0} \exp \left[-\left(z_{i} e \phi(z)+U_{i}(z)\right)\right]}{\epsilon_{0} \epsilon_{w}(0)}, \\
\frac{d \phi}{d z}_{z=0}=\frac{d \phi}{d z}{ }_{z=L}=0 .
\end{gathered}
$$

The ionic dispersion potentials are complemented with a hardsphere repulsion, which prevents all the ions from coming closer than one hardsphere radius of the ion from each surface. The above expression together with a particular salt concentration, water sheet thickness, $B_{j 2}$ values and the hardsphere ion radii are used to calculate the double layer contribution [35,

$$
\begin{gathered}
A(L)=\frac{e}{2} \int_{0}^{L} \phi \sum_{i} c_{i} z_{i} d z+\int_{0}^{L} \sum_{i} c_{i} U_{i} d z+ \\
\frac{1}{\beta} \int_{0}^{L} \sum_{i}\left[c_{i} \ln \left(c_{i} / c_{i, 0}\right)-\left(c_{i}-c_{i, 0}\right)\right] d z,
\end{gathered}
$$

where $A(L)$ is the free energy of two planar interfaces a distance $L$ apart, $\mathrm{c}_{i}$ is the concentration of ion $i$ at a specific point and $\mathrm{c}_{i, 0}$ the ion concentration in the bulk. The force is then calculated as $p(L)=-d A(L) / d L$.

In fig. 4, we show the ion-specific total force for two interfaces separated by $1 \mathrm{mM} \mathrm{NaCl}$ salt concentration in
Table 1: The $B_{12}$ values (the non-retarded van der Waals energy times $z^{3}$ in units of $10^{-50} \mathrm{~J} \mathrm{~m}^{3}$ ) for ion-surface interaction for the hardsphere and Onsager's models near different interfaces. For the ice-water interface, we give two sets of $B_{12}$ values using the ice $_{13}$ and ice in $_{12}$ (in parenthesis) models of dielectric function for ice 6 .

\begin{tabular}{cccc}
\hline \hline Interface & Ionic species & $B_{12}$ (Onsager) & $B_{12}$ (hardsphere) \\
\hline & $\mathrm{Cl}^{-}$ & $0.046(-0.026)$ & $0.19(0.18)$ \\
ice-water & $\mathrm{Br}^{-}$ & $0.088(0.0012)$ & $0.28(0.27)$ \\
& $\mathrm{I}^{-}$ & $0.19(0.089)$ & $0.48(0.47)$ \\
& $\mathrm{Na}^{+}$ & $-0.049(-0.069)$ & $-0.046(-0.065)$ \\
& $\mathrm{K}^{+}$ & $-0.12(-0.17)$ & $-0.081(-0.12)$ \\
\hline \multirow{5}{*}{ vapor-water } & $\mathrm{Cl}^{-}$ & 13.50 & 7.99 \\
& $\mathrm{Br}^{-}$ & 17.14 & 10.85 \\
& $\mathrm{I}^{-}$ & 23.49 & 16.47 \\
& $\mathrm{Na}^{+}$ & 0.82 & 0.69 \\
& $\mathrm{~K}^{+}$ & 3.83 & 3.45 \\
\hline & $\mathrm{Cl}^{-}$ & -5.70 & -3.56 \\
$\mathrm{CO}_{2}$-water & $\mathrm{Br}^{-}$ & -7.17 & -4.68 \\
& $\mathrm{I}^{-}$ & -9.67 & -6.72 \\
& $\mathrm{Na}^{+}$ & -0.45 & -0.40 \\
& $\mathrm{~K}^{+}$ & -1.87 & -1.7 \\
\hline \hline
\end{tabular}

water and the Lifshitz contribution to the total force as a function of distance across the salt water. Here we consider ice-water-vapor, ice-water- $\mathrm{CO}_{2}$, and vapor-water$\mathrm{CO}_{2}$ systems. For systems without salt, the double layer force is absent, and the force is given by the retarded Lifshitz force. The double-layer contribution correlates with the magnitude and sign of $\mathrm{B}_{12}$ values at the different interfaces. Both systems involving vapor-water interfaces are dominated by the rather large repulsive $\mathrm{B}_{12}$ values at this interface. The force changes sign at $2.1 \mathrm{~nm}$ for the ice $_{13}$-water-vapor system for Lifshitz interaction while no reversal of sign occurs for the double layer interaction. The sign does not reverse in the vapor-water- $\mathrm{CO}_{2}$ system for both Lifshitz and the double layer interactions. The ice-salt-water- $\mathrm{CO}_{2}$ system is quite unique with a sign reversal in the force for both the linear retarded Lifshitz term (at $5.3 \mathrm{~nm}$ ) and the non-linear van der Waals force from ion-surface interactions (at $4.4 \mathrm{~nm}$ ). For $1 \mathrm{mM} \mathrm{NaCl}$ concentration, we observe that the $\mathrm{CO}_{2}$ bubbles in water tend to get attracted to the ice surface when they come closer to the surface, while they are always repelled from the vapor surface. The two additional terms that also arise when we solve the Poisson-Boltzmann equation for the ion profiles under the influence of van der Waals forces and electrostatic forces are the entropic term and the electrostatic term. The electrostatic term is almost negligible in the present case, while the entropic term contributes to attraction for all separations but the behaviour is globally dominated by the non-linear van der Waals term. This term comes from ion-surface van der Waals force acting on the ions in the ion profile. Similar to the linear Lifshitz term it contributes to short range attraction and long range repulsion. This means that the $\mathrm{CO}_{2}$ bubbles that 


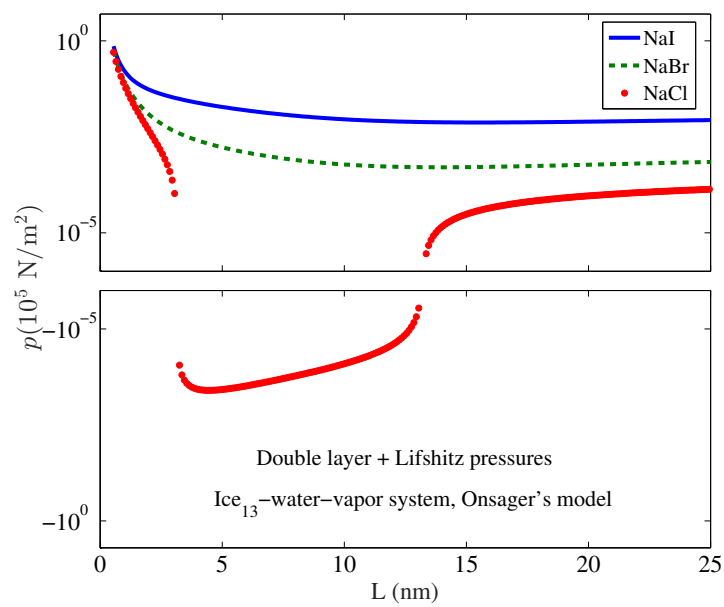

Fig. 5: (Color online) The force between ice and vapor interfaces separated by $1 \mathrm{mM}$ of different salt solutions as a function of water film thickness. We consider the ice 13 model for the dielectric function of ice and the Onsager model for effective polarizability of the ions in water.

come very close to the interface bind to the ice substrate by the combined effect of attractive linear and non-linear dispersion forces.

In fig. 5, we present the total force for the ice-watervapor system with a fixed concentration $(1 \mathrm{mM})$ of different salts in water using Onsager's model of effective polarizability. The contribution from gravity $(-\rho g L)$ is negligible for the distances considered here. The reversal of the sign of the force in ice-water-vapor system facilitates the formation of a thin film of water of finite thickness, otherwise also known as incomplete surface melting 6]. We observe from fig. 5 that reversal depends not only on the dielectric functions of the three layers, but also on the ionic species. For salts, like $\mathrm{NaBr}$ and $\mathrm{NaI}$ with higher values of positive van der Waals $B$ parameters, the double layer force is too repulsive in the case of Onsager's model so that there is no finite equilibrium water film thickness. $1 \mathrm{mM} \mathrm{NaBr}$ and $\mathrm{NaI}$ give repulsion for all water film thicknesses. In other words, with an unlimited reservoir of salt ions in water surface melting of ice is complete. If, however, there is a fixed amount of salt ions on the surface the salt concentration will go down when the water sheet thickens. This will stop the system from undergoing complete melting. An equilibrium thin film does appear for these ions with the hardsphere model.

In fig. 6, we show that the equilibrium thickness of water in the ice-water-vapor system increases with increasing concentration of $\mathrm{NaCl}$. The results change slightly with the application of different models of the dielectric function of ice. However, the change due to application of the different models of effective polarizability of salt ions is significant. As stated earlier, Onsager's model for effective polarizability gives faster growing water films than as given by the hardsphere model. With the hardsphere

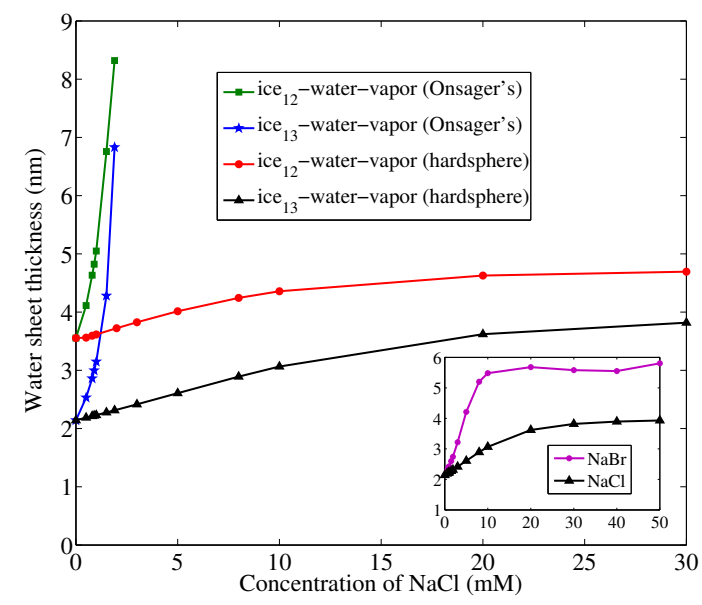

Fig. 6: (Color online) Equilibrium water sheet thickness for ice-water-vapor system as a function of $\mathrm{NaCl}$ salt concentration using different models for the dielectric function of ice and the polarizability of the ions. In the inset figure, we compare the equilibrium water film thickness for different salts using the hardsphere model and the ice ${ }_{13}$ model for the dielectric finction of ice.

model, the water film first grows with concentration and then, maintains a constant finite equilibrium thickness after a certain point. The equilibrium water film grows faster with increasing $\mathrm{NaBr}$ concentration than with increasing $\mathrm{NaCl}$ concentration (see inset of fig. 6.)

Our model neglects some microscopic aspects that are discussed in the literature on ice premelting 36] (and references therein), such as the molecular organization of liquid water at the interfaces (liquid-solid and liquid-vapor), that extends for 1-2 nm from the surface, the consequent change of dielectric constant there, and the effect of ions on them. For example, it is not always the case that ions have no specific adsorption behavior at an interface mediated by such molecular organization, and that this may be different for the positive and negative ions making the electrostatic potential different and the double-layer contribution to the thickness also different.

In this work, we have explored a simple model system where cold bubbles interact with ice-water or vapor-water interfaces. This enables us to study the trends when van der Waals and Lifshitz forces are included. The vital retardation effects have not been accounted for in molecular simulations on premelting (see for instance the work of Limmer and Chandler 36]). We have also investigated how the presence of salt ions influences the equilibrium thickness of the Casimir-induced water layer on ice surface. We predict an equilibrium water film thickness for ice-water-vapor system that increases with the increase in concentration of salt in the water phase. The results are directly applicable to the melting of ice under equilibrium conditions discussed by Elbaum and Schick 6]. We have found that the predictions strongly depend on the model 
employed for the effective polarizability of the ion in water. We have used the Onsager and hardsphere models. For this reason, a more realistic hybrid model as suggested in Ref. 34 should be developed in the future, and this model should be cross-compared with microscopic simulations based on density-functional theory. One should note that we are neglecting the chemical equilibrium of $\mathrm{CO}_{2}$ in water. This gas is not inert for water as it forms different hydrated species and ions. And its solubility depends on the ionic strength. This may very well influence the molecules and ion concentration at the interface and in solution. We plan to return to these interesting aspects in a later publication which will also include the effect of varying the $\mathrm{pH}$ in solution.

PT acknowledges support from the European Commission; this publication reflects the views only of the authors, and the Commission cannot be held responsible for any use which maybe made of the information contained therein. SYB gratefully acknowledges support by the German Research Foundation (grant BU 1803/3-1) and the Freiburg Institute for Advanced Studies. MB, OIM, and CP acknowledge support from the Research Council of Norway (Project: 221469). ERAL acknowledges support from the Brazilian agency FAPERJ. We acknowledge access to HPC resources at NSC through SNIC and at USIT through NOTUR. We thank Prof. B. W. Ninham for his comments on our manuscript.

\section{REFERENCES}

[1] ORR, F. M. Science 325 (2009) 1656.

[2] BENSON, S. M. and ORR, F. M. MRS Bull. 33 (2008) 303 .

[3] PIRES, J. C. M., MARTins, F. G., AlviM-FERRAZ, M. C. M. and SIMÕES, M. Chem. Eng. Res. Des. 89 (2011) 1446.

[4] ROCHELlE, C. A., CZERNICHOWSKI-LAURIOL, I. and MILODOWSKI, A. E. Geological Society, London, Special Publications 233 (2004) 87.

[5] TAKU IDE, S., JESSEN, K. and ORR JR, F. M. International Journal of Greenhouse Gas Control 1 (2007) 481.

[6] ELBAUM, M. and SCHICK, M. Phys. Rev. Lett. 66 (1991) 1713.

[7] NESHYBA, S., NUGENT, E., ROESELOVA, M. and JUNGWIRTH, P. J. Phys. Chem. C 113 (2009) 4597.

[8] MARTIN-TORRES, F. J. ET AL, Nature Geoscience 8 (2015) 357.

[9] DZYAlOSHINSKII, I. E., LIFSHITZ, E. M. and PITAEVSKII, P. P. Advan. Phys. 10 (1961) 165.

[10] RICHMOND, P., NINHAM, B. W. and OTTEWILL, R. H. J. Colloid Int. Sci. 45 (1973) 69.

[11] RICHMOND, P. and NINHAM, B. W. Solid State Communications 9 (1971) 1045.

[12] ANDERSON, C. H. and SABISKY, E. S. Phys. Rev. Lett. 24 (1970) 1049.

[13] HAUXWELL, F. and OTTEWILL, R. H. J. Colloid Int. Sci. 34 (1970) 473.

[14] NINHAM, B.W. and PARSEGIAN, V.A. Biophys. J. 10 (1970) 646 .
[15] DERJAGuiN, B. V., ABRIKOSOVA, I. I. and LIFSHITZ, E. M. Q. Rev. Chem. Soc. 10 (1956) 295 .

[16] NINHAM, B. W. and LO NOSTRO, P. Molecular Forces and Self Assembly: in Colloid, Nano Sciences and Biology, (Cambridge University Press, Cambridge, 2010).

[17] NINHAM, B.W. and YAMINSKY, V. Langmuir 13 (1997) 2097.

[18] WERNERSSON, E. and KJELLANDER R. J. Phys. Chem. B 111 (2007) 14279.

[19] BOSTRÖM, M., WILliAMS D. R. M. and NINHAM B. W. Phys. Rev. Lett. 87 (2001) 168103.

[20] MALYI, O. I., BOSTRÖM, M., KULISH, V. V., THIYAM, P., PARSONS, D. F., PERSSON, C. Phys. Chem. Chem. Phys. (submitted).

[21] KRUKAU, A. V., VYDROV, O. A., IZMAYLOV, A. F. and SCUSERIA, G. E. J. Chem. Phys. 125 (2006) 224106.

[22] SERNELIUS, BO E. Surface Modes in Physics (WileyVCH, Berlin, 2001).

[23] FRENCH, M. and REDMER, R. Phys. Plasmas 18 (2011) 043301.

[24] DANIELS, J. Opt. Commun. 3 (1971) 240.

[25] SEKI, M., KOBAYASHI, K. and NAKAHARA, J. J. Phys. Soc. Jpn. 50 (1981) 2643.

[26] BONNEFOY, O., GRUY, F., HERRI, J.-M. Fluid Phase Equilibria 231 (2005) 176.

[27] J. MAHANTY and B. W. NINHAM, Dispersion Forces, (Academic Press, London, 1976).

[28] SAMBALE, A., WELSCH, D.-G., TRUNG DUNG, HO and BUHMAnN, S. Y. Phys. Rev. A 79 (2009) 022903.

[29] ONSAGER, L. J. Am. Chem. Soc. 58 (1936) 1486.

[30] SAMBALE, A. , BUHMANN, S. Y. , WELSCH, D.-G. and TOMAŠ, M. S. Phys. Rev. A 75 (2007) 042109.

[31] PARSONS, D.F. and NINHAM, B. W. Langmuir 26 (2010) 1816.

[32] PARSOnS, D.F. and Ninham, B. W. J. Phys. Chem. A 113 (2009) 1141.

[33] JACKSON, J. D. Classical Electrodynamics (Wiley, New York, 1998).

[34] SAMBALE, A., BUHMANN, S. Y. and SCHEEL, S. Phys. Rev. A 81 (2010) 012509.

[35] LIMA, E. R. A., BOSTRÖM, M., SCHWIERZ, N., SERNELIUS, B. E. and TAVARES, F. W. Phys. Rev. E 84 (2011) 061903.

[36] LimMeR, D. T. and CHANDLER, D. J. Chem. Phys. 141 (2014) 18C505. 